\title{
Enhanced host-guest electrochemical recognition of herbicide MCPA using a $\beta$-cyclodextrin carbon nanotube sensor
}

\author{
V. Rahemi, J.J. Vandamme, J.M.P.J. Garrido, F. Borges, C.M.A. Brett, E.M.P.J. Garrido
}

\author{
A B S T R A C T
}

An electrochemical sensor for the determination of the chlorophenoxy herbicide MCPA has been developed, based on a combination of multi-walled carbon nanotubes with incorporated $\beta$-cyclodextrin and a polyaniline film modified glassy carbon electrode. The proposed molecular host-guest recognition based sensor has a high electrochemical sensitivity for the determination of MCPA. The electrochemical behaviour of MCPA at the chemically modified electrode was investigated in detail by cyclic voltammetry. The results indicate that the $\beta-C D / M W C N T$ modified glassy carbon electrode exhibits efficient electrocatalytic oxidation of MCPA with high sensitivity, stability and lifetime. The analytical characteristics of this film were used for the quantitative determination of MCPA in natural waters. Cyclic voltammetry in phosphate buffer solution at $\mathrm{pH} 6.0$, allowed the development of a method to determine MCPA, without any previous steps of extraction, clean-up, or derivatization, in the range of $10-100 \mu \mathrm{mol} \mathrm{L}^{-1}$, with a detection limit of $0.99 \mu \mathrm{mol} \mathrm{L}^{-1}$ in water. The results were statistically compared with those obtained through an established high-performance liquid chromatography technique, no significant differences having been found between the two methods.

\section{Keywords:}

Herbicides

MCPA

Voltammetric sensors

Modified electrodes

$\beta$-cyclodextrin

Carbon nanotubes

\section{Introduction}

Agricultural and industrial chemicals, pollutants and toxicants are now widely dispersed in the environment. Among them, pesticides are used extensively in agriculture and industry to enhance food production by eradicating unwanted insects and controlling disease vectors $[1,2]$. The presence of pesticide residues and metabolites in food, water and soil currently represents one of the major issues for environmental chemistry. In fact, these chemicals are known to have a cumulative hazardous effect on humans and animals; thus, regulations for drinking water are required in order to limit human risks and environmental pollution [3].

Phenoxy acid herbicides showing auxin-like activity have been extensively used to control the growth of grass and broad-leaf weeds in many crops, such as rice [4], winter wheat [5,6], soybean [7], etc. The chlorophenoxy herbicide MCPA (4-chloro-2-methylphenoxyacetic acid) is widely used for the control of broad-leaf weeds primarily in cereal and grass seed crops [8-10]. In Portugal,
MCPA still remains one of the most often-used herbicides for rice crops [11]. MCPA is not extensively metabolised and urine is the predominant route of excretion [12]. Although MCPA is only a moderately toxic herbicide, monitoring its residues in water and soil is crucial because it can influence the nervous system upon adsorption through the skin and its prolonged inhalation can cause dizziness, burning in the chest and coughing [13].

Contemporary methods for environmental determination and/ or the monitoring of pesticides include gas and liquid chromatography and various spectroscopic techniques [14]. Each of these approaches suffers from several disadvantages such as being costly, time-consuming, not sufficiently sensitive and/or requiring complex sample preparation. Moreover, continuous monitoring is not possible with any of these methods and it follows that a simplified analytical approach would prove highly beneficial.

A potential solution to this problem is the utilisation of electrochemical sensor technology. Electrochemical sensors can provide fast, reliable and cost-effective measurement and monitoring methods. The new demands of environmental analysis have driven the development of more selective and sensitive sensing systems and the advent of nanotechnology has led to great advances in detection strategies. Attractive properties of nanomaterials such as large surface to volume ratio, high packing density and long-range order may serve to be potentially useful in emerging environmental applications. Carbon nanotubes (CNTs) 
have generated great interest in applications based on their field emission and electronic transport properties, their high mechanical strength, high-surface to volume ratio and their chemical properties [15]. The combination of CNT electrocatalytic activity with the known advantages of other compounds, such as polymers, enzymes or macromolecules, can be a very important and new way for solving electroanalytical challenges.

The molecule $\beta$-cyclodextrin ( $\beta$-CD), a macrocyclic compound with seven D-glucopyranose units linked by a-1,4-glycosidic bonds which possesses a hydrophilic exterior and an interior hydrophobic cavity, has been extensively investigated in host-guest chemistry for the construction of versatile supramolecular aggregations owing to its special hydrophobic cavities and ability to improve the physico-chemical properties and chemical stability of drugs [16-18]. Electroanalytical applications of CDs are based on formation of an inclusion complex, molecular recognition and selective pre-concentration of analytes at the electrode [19].

Taking into consideration the electrochemical properties of CNTs as well as the reported properties of $\mathrm{CD}$, this work proposes a novel strategy based on the simultaneous modification of a glassy carbon electrode with a novel polyaniline-CNT cyclodextrin matrix (PANI$\beta-C D / M W C N T)$ for the determination of MCPA herbicide. The chemical recognition of MCPA by the use of $C D$ is combined with the added advantage of a faster electron transfer process due to the CNT present at the electrode interface. The modified electrode developed was employed for the direct oxidative determination of MCPA in pure and natural water samples by cyclic voltammetry.

\section{Experimental}

\subsection{Reagents}

Multi-walled carbon nanotubes (MWCNTs) were obtained from NanoLab (USA). MCPA, aniline and $\beta$-cyclodextrin $(\beta-C D)$ were supplied by Sigma-Aldrich Química (Sintra, Portugal). Analytical grade reagents purchased from Merck (Darmstadt, Germany) were used without additional purification.

Double distilled deionized water was used throughout the experiments. Buffer solutions employed for voltammetric determinations were $0.1 \mathrm{M}$ in the $\mathrm{pH}$ range $1.2-12.2$.

HPLC-grade acetonitrile was obtained from Carlo Erba. Prior to use, the solvents were filtered through a $0.45-\mu \mathrm{m}$ filter.

\subsection{Apparatus}

Voltammetric experiments were performed using an Autolab PGSTAT 12 potentiostat/galvanostat (EcoChemie, Netherlands) in a one-compartment glass electrochemical cell equipped with a threeelectrode system consisting of a bare or modified glassy carbon working electrode (GCE, $d=2 \mathrm{~mm}$ ), a platinum wire counter electrode and an $\mathrm{Ag} / \mathrm{AgCl}$ (saturated $\mathrm{KCl}$ ) reference electrode. All measurements were carried out at room temperature.

A Crison $\mathrm{pH}$-meter with glass electrode was used for the $\mathrm{pH}$ measurements (Crison, Spain).

The HPLC method [20] was performed using a HPLC/DAD system consisting in a Shimadzu instrument (pumps model LC20AD, Tokyo, Japan), equipped with a commercially prepacked Nucleosil 100-5 C18, analytical column $(250 \mathrm{~mm} \times 4.6 \mathrm{~mm}, 5 \mu \mathrm{m}$, Macherey-Nagel, Duren, Germany) and UV detection (SPD-M20A) at the wavelength maximum determined by analysis of the UV spectrum $(234 \mathrm{~nm})$. The mobile phase consisted of $43.7 \mathrm{mM}$ acetic acid at pH 2.5 with $40 \%(\mathrm{v} / \mathrm{v})$ acetonitrile. It was delivered isocratically at $1 \mathrm{~mL} \mathrm{~min}^{-1}$ at room temperature.

The chromatographic data was processed in a Samsung computer, fitted with LabSolutions software (Shimadzu, Japan).

\subsection{Preparation of modified electrochemical sensors}

Multi-walled carbon nanotubes (MWCNTs) were purified and functionalised as described elsewhere [21]. A mass of $120 \mathrm{mg}$ of MWCNTs was stirred in $10 \mathrm{~mL}$ of a $5 \mathrm{~mol} \mathrm{~L}^{-1}$ nitric acid solution for $20 \mathrm{~h}$. The solid product was collected on a filter paper and washed several times with double-distilled water until the filtrate solution became neutral $(\mathrm{pH} \approx 7)$. The functionalised MWCNTs (fMWCNT) obtained were then dried in an oven at $80^{\circ} \mathrm{C}$ for $24 \mathrm{~h}$. Nitric acid usually causes the significant destruction of carbon nanotubes and introduces - $\mathrm{COOH}$ groups at the ends or at the sidewall defects of the nanotube structure.

Two milligrams of MWCNTs or fMWCNTs was dispersed by using ultrasonic agitation in $1 \mathrm{~mL}$ aqueous $\beta$-CD solution (2\%) to give a $2 \mathrm{mg} \mathrm{mL}^{-1}$ black suspension.

Prior to use, the $2 \mathrm{~mm}$ GC working electrode was carefully polished with an aqueous slurry of alumina powder (BDH) on a microcloth pad and then thoroughly washed with double-distilled water. The electrode was then placed first in ethanol followed by double-distilled water, in each case being subjected to sonication to remove traces of alumina and possible contaminants. The clean GCE was then immersed in a solution containing $0.011 \mathrm{~mol} \mathrm{~L}^{-1}$ aniline and $0.025 \mathrm{~mol} \mathrm{~L}^{-1} \mathrm{H}_{2} \mathrm{SO}_{4}$ and the potential was swept between -0.1 and $1.0 \mathrm{~V}$ vs. $\mathrm{Ag} / \mathrm{AgCl}$ at a scan rate of $50 \mathrm{mV} \mathrm{s}^{-1}$ for 50 cycles. After preparation of the polyaniline film on the GC electrode surface, an aliquot of $6 \mu \mathrm{L}$ of the $2 \mathrm{mg} \mathrm{mL}^{-1}$ of the previously-prepared suspension of MWCNTs or fMWNTs was dropped onto the electrode surface and dried in air at room temperature.

\subsection{Analytical procedure}

The PANI- $\beta-C D / f M W C N T$ film coated GC sensor was first activated in phosphate buffer $(\mathrm{pH} 6)$ by cyclic voltammetric sweeps between 0.5 and $1.3 \mathrm{~V}$ until stable cyclic voltammograms were obtained. Accurate volumes of the stock standard solution of MCPA (10 mM) were then added to the voltammetric cell and CVs were recorded from 0.5 to $1.3 \mathrm{~V}$ at a scan rate of $50 \mathrm{mV} \mathrm{s}^{-1}$. The same procedure was applied for the analysis of natural water samples. After every measurement, the PANI- $\beta-\mathrm{CD} / \mathrm{fMWCNT}$ film coated GC sensor was cleaned by successive cyclic voltammetric sweeps in $0.1 \mathrm{M}$ phosphate buffer $(\mathrm{pH} 6)$ solution until unchanged cyclic voltammograms were obtained.

\subsection{Sample preparation}

A water sample from Leça river (a polluted river with a high content of organic components) was collected in 2.5-L brown glass bottles. Immediately after arrival in the laboratory, the samples were filtered through $1-\mu \mathrm{m}$ glass fibre filters and $0.45-\mu \mathrm{m}$ cellulose acetate filters, sequentially, to remove suspended particles.

\subsection{Analysis of MCPA in water samples}

Standard stock solutions of MCPA (10 mM) were prepared in ethanol. For calibration curves, standard solutions were prepared in the voltammetric cell by adding accurate volumes of the stock standard solution of MCPA to the selected phosphate pH 6 supporting electrolyte in order to obtain concentrations between 10 and $100 \mu \mathrm{M}$. The calibration curve for CV analysis was constructed by plotting the peak current against the MCPA concentration $(10,40,60,80$ and $100 \mu \mathrm{M})$. The limit of quantification (LOQ) and the limit of detection (LOD) were calculated according to IUPAC recommendations [22], using an $\mathrm{S} / \mathrm{N}$ ratio of ten and three, respectively. Method precision was checked on different days, within day $(n=5)$ and between days $(n=5)$ for three different concentrations. The accuracy of the proposed method 
was determined by recovery assays and comparing the results with those obtained from a previously-published HPLC method [20]. Recovery assays were carried out at different concentrations by adding known amounts of standard solution of MCPA to the river water samples. The final concentrations of MCPA, after this addition, were 10,40 and $60 \mu \mathrm{M}$.

\section{Results and discussion}

\subsection{Characterisation of the modified electrodes}

Previous reports have already discussed the interfacial architecture and electrochemical activity of CNT-modified electrodes after incorporating CDs and recognition effects [23,24]. Thus, electrochemical studies have been carried out to investigate the enhancement effect of $\beta$-CD/MWCNT composites with PANI on glassy carbon electrodes (GCE) as substrates. The PANI/MWCNT film coated GC electrodes, both unmodified and modified with $\beta-C D$, were characterised by cyclic voltammetry in the presence of $1 \mathrm{mM}$ potassium ferricyanide as electroactive species (Fig. 1).

The electroactive areas of the modified GC electrodes were calculated using cyclic voltammetry and compared to that of the bare GC electrode. In all cases, at bare or modified electrodes, the peak current increases linearly with the square root of scan rate for both oxidation and reduction, consistent with a diffusioncontrolled process. Based on the Randles-Sevcik equation $I_{\mathrm{p}}=2.69 \times 10^{5} \mathrm{n}^{3 / 2} A D_{0}^{1 / 2} C_{0} v^{1 / 2}$, the electroactive area, $A$, can be determined from the slope of a plot of the voltammetric peak current, $I_{\mathrm{p}}$, vs. the square root of scan rate, $v^{1 / 2}$ [25]. From the slopes of the straight lines obtained for the bare electrode, PANI/MWCNT/GCE, PANI- $\beta-C D / M W C N T / G C E$ and PANI- $\beta-C D / f M W C N T / G C E$, the electroactive surface areas of the corresponding electrodes are calculated to be $0.046,0.117,0.131$ and $0.146 \mathrm{~cm}^{2}$, respectively. The PANI- $\beta$ $\mathrm{CD} / \mathrm{fMWCNT/GC}$ electrode shows around a 3-fold increase in the electroactive surface area compared with the bare GC electrode, which will lead to a more sensitive response to the analyte.

\subsection{Electrochemistry of MCPA at PANI- $\beta-C D / M W C N T$ GC electrodes}

A study of MCPA electro-oxidation was carried out by cyclic voltammetry, scanning the potential from +0.5 to $+1.3 \mathrm{~V}$ in

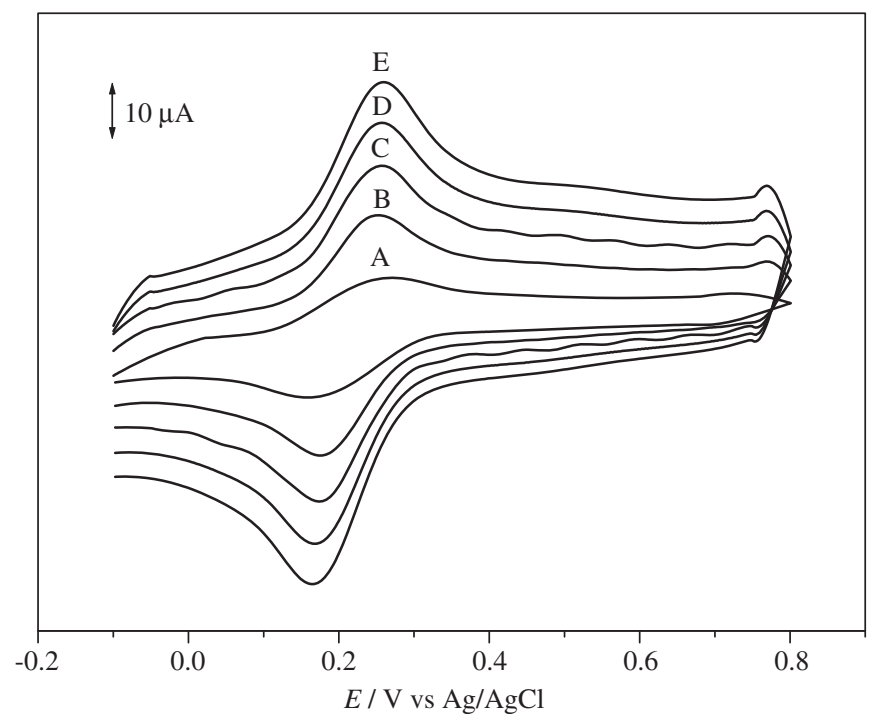

Fig. 1. Cyclic voltammograms in $1 \mathrm{mM} \mathrm{K}_{3}\left[\mathrm{Fe}(\mathrm{CN})_{6}\right] / 0.1 \mathrm{M} \mathrm{KCl}$ at PANI- $\beta$ CD/fMWCNT/GCE at different scan rates: (A) 20, (B) 40, (C) 60, (D) 80 and (E) $100 \mathrm{mV} \mathrm{s}^{-1}$

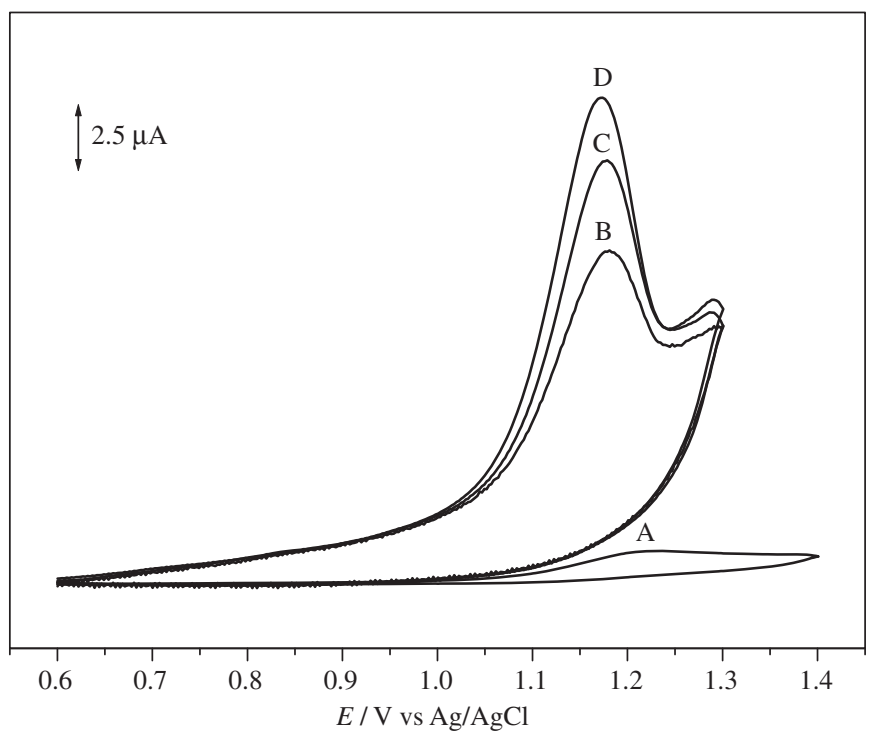

Fig. 2. Cyclic voltammograms of $0.1 \mathrm{mM}$ solutions of MCPA at (A) GCE, (B) PANI/ MWCNT/GCE, (C) PANI- $\beta-C D / M W C N T / G C E$ and (D) PANI- $\beta-C D / f M W C N T / G C E$ in pH 6 phosphate buffer electrolyte. Scan rate: $50 \mathrm{mV} \mathrm{s}^{-1}$.

$0.1 \mathrm{~mol} \mathrm{~L}^{-1}$ phosphate buffer solution ( $\mathrm{pH} \mathrm{6.0),} \mathrm{containing} 100 \mu \mathrm{M}$ of MCPA. Fig. 2 shows MCPA cyclic voltammograms obtained using a scan rate of $50 \mathrm{mV} \mathrm{s}^{-1}$ at the bare GC electrode (curve A), at the PANI/MWCNT (curve B), at the PANI- $\beta-C D / M W C N T$ (curve $C$ ) and PANI- $\beta-C D / f M W C N T$ (curve D). The results obtained for all four types of electrode demonstrate that, in the potential range studied, there is only one oxidation wave with no corresponding reduction on scan reversal, suggesting that the electrochemical reaction is a totally irreversible process. The electrochemical response of MCPA at the bare GC electrode is rather poor (curve A), with a broad and small anodic peak at $1.213 \mathrm{~V}$. When the GC electrode was modified with the PANI/MWCNTs film, the oxidation of MCPA occurred at a less positive potential (potentials shifted negatively by about $40 \mathrm{mV}$ ) and the peak current increased substantially, showing the electrocatalytic effect of the CNTs. Moreover, the use of functionalised MWCNTs slightly enhanced the peak current, which can be related to the increase of the surface-active area of the carbon nanotubes. The addition of $\beta-C D$ to the film-modified GC electrodes led to a significant increase in the peak currents.

The significant increase of the current for the PANI- $\beta-C D /$ MWCNTs GC electrodes compared to the bare GCE can be explained considering that the apparent concentration of MCPA- $\beta-C D$ in the PANI- $\beta-C D / M W C N T$ film is much higher due to the $\beta-C D$ encapsulation effect of MCPA [26]. The MCPA- $\beta-C D$ complex in PANI- $\beta-C D /$ MWCNT then dissociates and diffuses rapidly through the porous layer of MWCNT to the GC surface. The increase in current is also intimately related with the ability of MWCNT to mediate electrontransfer reactions with electroactive species present in solution. These phenomena demonstrate that PANI- $\beta-C D / M W C N T / G C E$ not only shows the advantage of MWCNT, but also that of organic molecules interacting with $\beta-C D$.

\subsection{Influence of $\mathrm{pH}$ and scan rate}

In order to investigate any influence of the $\mathrm{pH}$ of the buffer solution on the electrode reaction, the effect of different $\mathrm{pH}$ electrolytes on the current response was investigated by cyclic voltammetry (Fig. 3).

The anodic peak potential was shifted to more negative values as the $\mathrm{pH}$ increased from 1 to 4 (Fig. 3). The $E_{\mathrm{p}}-\mathrm{pH}$ plot shows that the electrode process is $\mathrm{pH}$-dependent; the slope of the line 


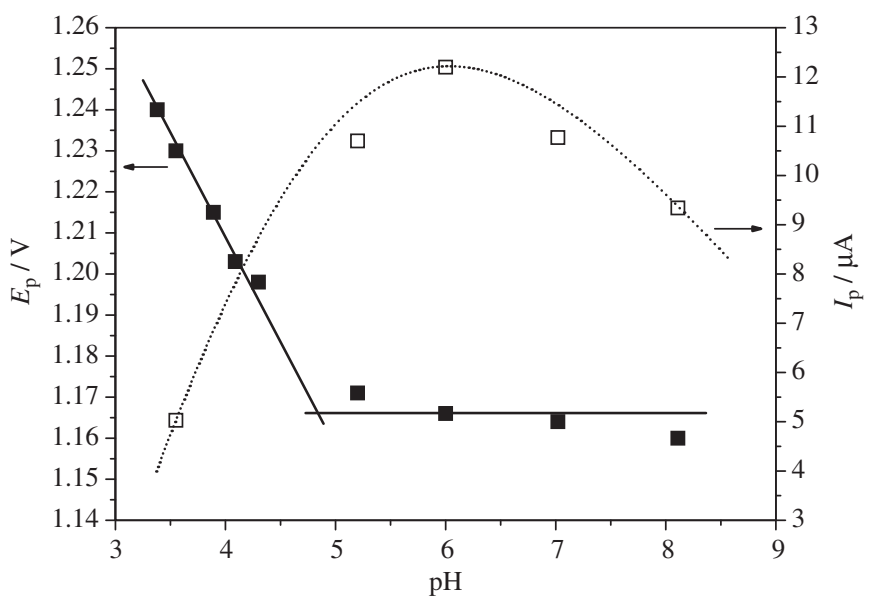

Fig. 3. Plots of $E_{\mathrm{p}}$ (filled symbols) and $I_{\mathrm{p}}$ (open symbols) vs. $\mathrm{pH}$ from cyclic voltammograms of $0.1 \mathrm{mM}$ solutions of MCPA at PANI- $\beta-C D / f M W C N T$ film electrode in different buffer electrolytes as a function of $\mathrm{pH}$. Scan rate: $50 \mathrm{mV} \mathrm{s}^{-1}$.

(Fig. 3), ca. $51 \mathrm{mV}$ per $\mathrm{pH}$ unit, suggesting that the reaction mechanism of this oxidation process in aqueous media involves the same number of electrons and protons. Previous electrochemical experiments showed that the oxidation process proceeds at the aromatic nucleus, through an electron transfer, via the formation of a cation radical intermediate that undergoes further reaction, namely the cleavage of the ether linkage (unpublished results). For $\mathrm{pH}$ values between 4 and 8 , no significant variation in the peak potential was observed. For $\mathrm{pH}$ values higher than 8 , a broad and poorly defined peak was seen.

The effect of the solution $\mathrm{pH}$ on the peak current was also assessed. A plot of $I_{\mathrm{p}}$ vs. $\mathrm{pH}$ indicates that the peak current reaches a maximum around $\mathrm{pH} 6$ (Fig. 3). Consequently, this buffer was chosen as the best supporting electrolyte and was used throughout the electroanalytical study.

The effect of scan rate on the peak current of MCPA was investigated in $\mathrm{pH} 6$ phosphate buffer solution. Fig. 4 shows MCPA voltammograms obtained at PANI- $\beta-C D / f M W C N T$ at different scan rates. The oxidation peak potential shifts with increasing scan rate toward more positive values, by about $70 \mathrm{mV}$ on going from 20 to $100 \mathrm{mV} \mathrm{s}^{-1}$, demonstrating some kinetic limitation of the electrochemical reaction. The peak current increases linearly with square root of scan rate according to the equation $I$ $(\mu \mathrm{A})=4.6755 v^{1 / 2}-5.782$ with a linear correlation coefficient of 0.995 . Therefore, the oxidation process of MCPA at the modified electrode is diffusion-controlled.

\subsection{Analytical application}

Experiments with differential pulse voltammetry did not produce a significant improvement in the sensitivity (increase of around 8\%). Considering the need of a CV cleaning step of the electrode surface between each experiment, it was found preferable to also use cyclic voltammetry as the detection method for the determination of MCPA. CV experiments were carried out in triplicate using the optimised experimental parameters to obtain an analytical curve for the determination of MCPA at the PANI- $\beta$ $\mathrm{CD} / \mathrm{fMWCNT}$ film electrode.

Cyclic voltammograms recorded with increasing amounts of MCPA $(10-100 \mu \mathrm{M})$ showed that the peak currents increased linearly with increasing concentration (Fig. 5). Analytical data for the calibration graphs are summarised in Table 1 . The limit of detection (LOD) and the limit of quantification (LOQ), obtained from five runs, are also presented in Table 1 . The precision of the method was evaluated by repeatedly $(n=5)$ measuring MCPA, at

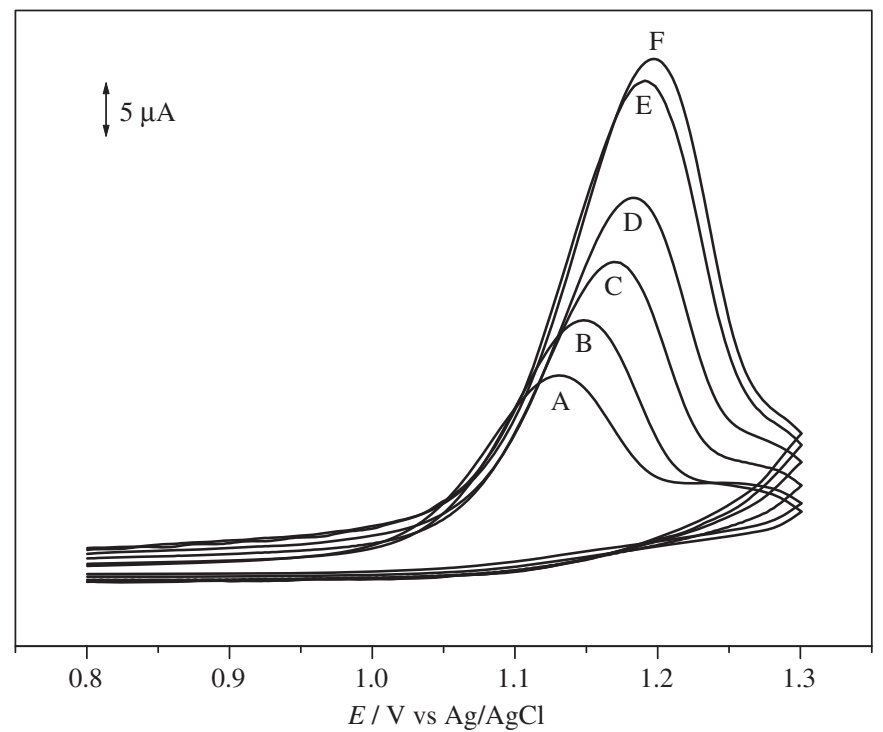

Fig. 4. Cyclic voltammograms of $0.1 \mathrm{mM}$ solution of MCPA at PANI- $\beta-\mathrm{CD} / \mathrm{fMWCNT}$ film electrode in $\mathrm{pH} 6$ phosphate buffer electrolyte at different scan rates: (A) 20 , (B) 30, (C) 50, (D) 70, (E) 90 and (F) $100 \mathrm{mV} \mathrm{s}^{-1}$.

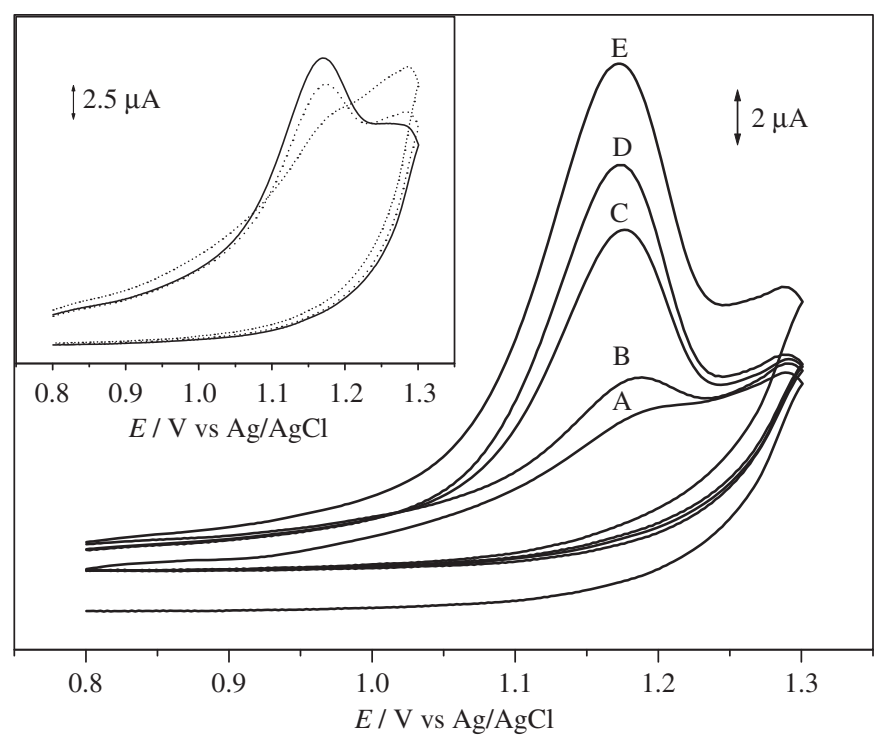

Fig. 5. Cyclic voltammograms of MCPA standard solutions with MCPA concentrations of: $A-10, B-40, C-60, D-80$ and $E-100 \mu \mathrm{M}$ at PANI- $\beta-C D / f M W C N T$ film electrode in $\mathrm{pH} 6$ phosphate buffer electrolyte. Inset: Cyclic voltammograms obtained for the determination of MCPA at a concentration of $(\bullet \bullet \bullet) 10 ;(\boldsymbol{\square} \mathbf{\square}) 40$ and (-) $60 \mu \mathrm{M}$ in spiked river water samples. Scan rate: $50 \mathrm{mV} \mathrm{s}^{-1}$.

three levels of concentration $(10,40$ and $60 \mu \mathrm{M})$ on the same day and over five consecutive days (Table 1 ).

The proposed method was applied to the analysis of the pesticide in river water spiked with MCPA, using the standard addition method, in order to eliminate any matrix effects. For this purpose, a water sample was spiked with MCPA in order to achieve final concentrations of 10,40 and $60 \mu \mathrm{mol} / \mathrm{L}$

Cyclic voltammograms obtained from the determination of MCPA in river water samples are presented in Fig. 5. The recoveries obtained were close to $100 \%$, as shown in Table 2 .

The accuracy of the voltammetric method proposed was assessed by comparing the results found with those obtained using 
Table 1

Linearity range, quantification and detection limits, inter-day repeatability for determination of MCPA, using the cyclic voltammetric method (CV), in $\mathrm{pH}$ 6 phosphate buffer electrolyte and river water samples.

\begin{tabular}{lll}
\hline & \multicolumn{2}{l}{ CV } \\
\cline { 2 - 3 } & Supporting electrolyte & River water \\
\hline Linearity range $(\mu \mathrm{M})$ & $10-100$ & $10-60$ \\
Calibration plot $(n=6)$ & & \\
Intercept $(\mu \mathrm{A})$ & 0.243 & 0.219 \\
Slope $\left(\mathrm{A} \mathrm{M}^{-1}\right)$ & 0.121 & 0.116 \\
Correlation coefficient & 0.995 & 0.994 \\
Standard error of intercept & 0.197 & 0.178 \\
Standard error of slope & 0.012 & 0.008 \\
Inter-day precision $(n=5)^{\mathrm{a}}$ & & \\
$10 \mu \mathrm{M}$ & 2.41 & 2.46 \\
$40 \mu \mathrm{M}$ & 1.64 & 2.81 \\
$60 \mu \mathrm{M}$ & 1.88 & - \\
LOD $(\mu \mathrm{M})$ & 0.99 & - \\
LOQ $(\mu \mathrm{M})$ & 3.3 &
\end{tabular}

a Precision as coefficient of variation $(\mathrm{CV}, \%)=$ standard deviation divided by mean measured concentration $\times 100$.

Table 2

Results obtained for the analysis of MCPA in river water samples using the proposed cyclic voltammetric method and HPLC.

\begin{tabular}{lllllll}
\hline Method & $\begin{array}{l}\text { MCPA } \\
\text { added } \\
(\mu \mathrm{M})\end{array}$ & $\begin{array}{l}\text { MCPA } \\
\text { found } \\
(\mu \mathrm{M})\end{array}$ & $\begin{array}{l}\text { Recovery } \\
(\%)^{\mathrm{a}}\end{array}$ & $\begin{array}{l}\text { RSD } \\
(\%)\end{array}$ & t-test $^{\mathrm{b}}$ & $F_{\text {-test }}{ }^{\mathrm{b}}$ \\
\hline $\mathrm{CV}$ & 10 & 10.2 & $102.0 \pm 2.3$ & 2.5 & 2.04 & 1.93 \\
& 40 & 38.0 & $99.5 \pm 1.7$ & 2.1 & 1.26 & 2.16 \\
& 60 & 59.0 & $100.3 \pm 2.1$ & 2.3 & 1.69 & 1.91 \\
HPLC & 10 & 11.0 & $105.6 \pm 3.2$ & 2.8 & & \\
& 40 & 41.0 & $101.2 \pm 2.5$ & 1.9 & & \\
& 60 & 58.0 & $97.6 \pm 2.9$ & 1.7 & & \\
\end{tabular}

a Average of five replicate measurements.

b Tabulated $t$ - and $F$-values, at $P=0.05$, are 2.31 and 6.39 , respectively.

a previously-published chromatographic procedure [20]. For both the CV and HPLC methods, five independent measurements were carried out. These results, summarised in Table 2, show that the data from the CV method agree well with those from chromatography. The $F$ - and $t$-test were carried out on the data and the validity of the results obtained was statistically examined. At a confidence level of $95 \%$, the values from the $t$ - and $F$-tests were less than the theoretical values, showing that there is no significant difference between the proposed CV technique and the HPLC method.

The results reveal that both methodologies have comparable precision and accuracy. However, for the PANI- $\beta-C D /$ fMWCNT film electrode, high sensitivity, good reproducibility and simple instrumentation, preparation and analytical procedure are important advantages.

The only electroanalytical determination of MCPA found in the literature tests $\mathrm{MnO}_{2}$-modified carbon paste electrodes and $\mathrm{Mn}$ modified glassy carbon electrodes, of which the former are better [27]. They obtained a detection limit of $0.97 \mu \mathrm{M}$, very close to the $0.99 \mu \mathrm{M}$ found here at the PANI- $\beta-\mathrm{CD} / \mathrm{fMWCNT}$ film electrode; the analytical sensitivity of $0.0327 \mathrm{~A} \mathrm{M}^{-1}$ cannot be directly compared since the electrode area is not specified in [27], but is lower by a factor of around 4 than that here, if electrode areas are the same or similar.

\subsection{Operational lifetime and selectivity}

The stability of the PANI- $\beta-\mathrm{CD} / \mathrm{fMWCNT/GC}$ modified electrode was tested over a 5-day period. Cyclic voltammetry of
MCPA at the modified electrode showed that the oxidation peak potential remained unchanged, and the initial anodic peak current was maintained during this period, with a relative standard deviation lower than $3.2 \%$. Voltammetric measurements carried out with different sensors showed that there is good reproducibility; the relative standard deviation for 9 electrodes prepared on different days was $3.75 \%$.

The influence of various substances as compounds that can potentially interfere with the determination of MCPA was studied. As interfering species, some ions (e.g. $\mathrm{Ca}^{2+}, \mathrm{Mg}^{2+}, \mathrm{NO}_{3}^{-}, \mathrm{PO}_{4}^{3-}$ ), 4-chloro-2-methylphenol (MCPA's main degradation product) and another commonly used phenoxyacetic herbicide, 2,4-D, were selected. At MCPA mass ratio 1:1, no significant interference was observed (signal change less than $5 \%$ ).

\section{Conclusions}

In this work a new electroanalytical procedure, based on the incorporation of $\beta$-cyclodextrin into multi-walled carbon nanotube and polyaniline film modified glassy carbon electrodes, is proposed for the monitoring of MCPA in natural waters, not requiring separations, clean-up or derivatization steps, which are indispensable in a number of other analytical methodologies. The strategy of using a modified glassy carbon electrode (GCE) using $\beta$-cyclodextrin as molecular receptor and MWCNT as enhancer of electron transfer improves the overall electrochemical response of the MCPA detection system.

The application of the analytical methodology to samples prepared with contaminated water as complex matrix showed that contamination with organic matter hardly influences the determination. Moreover, the results show that the new method is sensitive and does not lead to statistically significant differences compared to established HPLC methodology. This demonstrates that the electrochemical sensor described here can be used to analyse and monitor MCPA in real samples.

The use of a combination of host-guest electrostatic interactions with the catalytic and electronic properties of MWCNT provides a new way of constructing electrodes for environmental monitoring, with application for the reliable determination of pesticide residues. Further applications can be envisaged.

\section{Acknowledgement}

Financial support from Fundação para a Ciência e Tecnologia FCT/MCTES project PTDC/AGR-AAM/105044/2008, National Funds PIDDAC also co-financed by the European Community Fund FEDER through COMPETE-Programa Operacional Factores de Competitividade (POFC), is gratefully acknowledged.

\section{References}

[1] F.P. Carvalho, Environ. Sci. Policy 9 (2006) 685-692.

[2] J. Cooper, H. Dobson, Crop Prot. 26 (2007) 1337-1348.

[3] T. Katagi, in: D.M. Whitacre (Ed.), Reviews of Environmental Contamination and Toxicology, vol. 204, Springer Science, New York, 2010, pp. 1-132.

[4] F. Leganés, E. Fernández-Valiente, Arch. Environ. Contam. Toxicol. 22 (1992) 130-134.

[5] A.G. Ogg Jr., F.L. Young, Weed Technol. 5 (1991) 291-296.

[6] D.C. Heering, T.F. Peeper, Weed Technol. 5 (1991) 317-320.

[7] A.C. York, J.W. Wilcut, M.M. Keene, F.R. Walls Jr., Weed Technol. 5 (1991) 43-47.

[8] S.K. Mortensen, C.S. Jacobsen, Environ. Sci. Technol. 38 (2004) 6625-6632.

[9] C.W. Thorstensen, A. Christiansen, J. Agric. Food Chem. 49 (2001) 4199-4202

[10] C.D.S. Tomlin, The e-Pesticide Manual, 12th edition CD-ROM form, version 2.0, British Crop Protection Council, Hampshire, UK, 2001.

[11] Eurostat, The Use of Plant Protection Products in the European Union: Data 1992-2003, Office for Official Publications of the European Communities, Luxembourg, 2007. 
[12] B. van Ravenzwaay, G. Pigott, E. Leibold, Food Chem. Toxicol. 42 (2004) $115-125$.

[13] E. Hiller, S. Čerňanský, L. Zemanová, Pol. J. Environ. Stud. 19 (2010) 315-321.

[14] C. Aprea, C. Colosio, T. Mammone, C. Minoia, M. Maroni, J. Chromatogr. B: Anal. Tech. Biomed. Life Sci. 769 (2002) 191-219.

[15] M.A. Goicolea, A. Gómez-Caballero, R.J. Barrio, Pesticides-Strategies for Pesticides Analysis, in: M. Stoytcheva (Ed.), InTech, Rijeka, Croatia, 2011, pp. 333-358.

[16] M. Singh, R. Sharma, U.C. Banerjee, Biotechnol. Adv. 20 (2002) 341-359.

[17] J. Szejtli, Pure Appl. Chem. 76 (2004) 1825-1845.

[18] E.M. Martin Del Valle, Process Biochem. 39 (2004) 1033-1046.

19] A. Ferancová, J. Labuda, Fresenius J. Anal. Chem. 370 (2001) 1-10.

[20] A. Jankowska, M. Biesaga, P. Drzewicz, M. Trojanowicz, K. Pyrzynska, Water Res. 38 (2004) 3259-3264.
[21] C. Gouveia-Caridade, R. Pauliukaite, C.M.A. Brett, Electrochim. Acta 53 (2008) 6732-6739.

[22] J. Mocak, A.M. Bond, S. Mitchell, G. Scollary, Pure Appl. Chem. 69 (1997) 297-328.

[23] T. Yin, W. Wei, J. Zeng, Anal. Bioanal. Chem. 386 (2006) 2087-2094.

[24] Q. Shen, X. Wang, J. Electroanal. Chem. 632 (2009) 149-153.

[25] A.J. Bard, L.R. Faulkner, Electrochemical Methods: Fundamentals and Applications, John Wiley \& Sons, Inc., New York, 2001.

[26] E.M. Garrido, M. Santos, P. Silva, F. Cagide, J. Garrido, F. Borges, J. Environ. Sci. Health, Part B, http://dx.doi.org/10.1080/03601234.2012.693867, in press.

[27] N. Priyantha, A. Navaratne, S. Weliwegamage, C.B. Ekanayake, Int. J. Electrochem. Sci. 2 (2007) 433-443. 